\section{OPEN ACCESS}

Edited by:

Alberto Gullo,

Garibaldi Hospital, Italy

Reviewed by:

Yuebang Yin,

Nankai University, China

Haner Direskeneli,

Marmara University, Turkey

*Correspondence:

Snežna Sodin-Šemr

ssodin1@yahoo.com

Specialty section:

This article was submitted to

Rheumatology

a section of the journal

Frontiers in Medicine

Received: 01 December 2021 Accepted: 28 December 2021

Published: 20 January 2022

Citation:

Kuret T, Frank-Bertoncelj M, Lakota K,

Žigon P, Thallinger GG, Kopitar AN,

Čučnik S, Tomšič M, Hočevar A and

Sodin-Šemrl S (2022) From Active to

Non-active Giant Cell Arteritis:

Longitudinal Monitoring of Patients on

Glucocorticoid Therapy in

Combination With Leflunomide.

Front. Med. 8:827095.

doi: 10.3389/fmed.2021.827095

\title{
From Active to Non-active Giant Cell Arteritis: Longitudinal Monitoring of Patients on Glucocorticoid Therapy in Combination With Leflunomide
}

\begin{abstract}
Tadeja Kuret 1,2,3, Mojca Frank-Bertoncelj ${ }^{4}$, Katja Lakota ${ }^{2,3}$, Polona Žigon ${ }^{2,3}$, Gerhard G. Thallinger ${ }^{5,6}$, Andreja N. Kopitar ${ }^{7}$, Saša Čučnik ${ }^{2,8}$, Matija Tomšič², Alojzija Hočevar ${ }^{2}$ and Snežna Sodin-Šemrl ${ }^{2,3 *}$
\end{abstract}

\begin{abstract}
${ }^{1}$ Institute of Cell Biology, Faculty of Medicine, University of Ljubljana, Ljubljana, Slovenia, ${ }^{2}$ Department of Rheumatology, University Medical Centre Ljubljana, Ljubljana, Slovenia, ${ }^{3}$ Faculty of Mathematics, Natural Science and Information Technologies, University of Primorska, Koper, Slovenia, ${ }^{4}$ BioMed X Institute, Heidelberg, Germany, ${ }^{5}$ Institute for Biomedical Informatics, Graz University of Technology, Graz, Austria, ${ }^{6}$ OMICS Center Graz, BioTechMed Graz, Graz, Austria, ${ }^{7}$ Institute of Microbiology and Immunology, Faculty of Medicine, University of Ljubljana, Ljubljana, Slovenia, ${ }^{8}$ Faculty of Pharmacy, University of Ljubljana, Ljubljana, Slovenia
\end{abstract}

In the present study, we longitudinally monitored leukocyte subsets, expression of neutrophil surface adhesion molecules (CD62L and CD11b) and serum analytes in therapy-naïve patients with active giant cell arteritis (GCA). We collected blood samples at the baseline, and at weeks 1, 4, 12, 24, and 48 of follow-up, and evaluated short- and long-term effects of glucocorticoids (GC) vs. GC and leflunomide. Our aim was to identify candidate biomarkers that could be used to monitor disease activity and predict an increased risk of a relapse. Following high doses of GC, the numbers of CD4+ T-lymphocytes and B-lymphocytes transiently increased and then subsided when GC dose tapering started at week 4. In contrast, the numbers of neutrophils significantly increased during the follow-up time of 12 weeks compared to pre-treatment time. Neutrophil CD62L rapidly diminished after initiation of GC therapy, however its expression remained low at week 48, only in patients under combinatorial therapy with leflunomide. Levels of acute phase reactant SAA and IL-6 decreased significantly after treatment with $\mathrm{GC}$ and leflunomide, while levels of $\mathrm{LL}-8, \mathrm{IL}-18$, and $\mathrm{CH} 3 \mathrm{~L} \mathrm{~L} 1$ did not change significantly during the follow-up period. $\mathrm{CHI} 3 \mathrm{~L} 1$ was associated with signs of transmural inflammation and vessel occlusion and might therefore serve as a marker of fully developed active GCA, and a promising therapeutic target. Patients with relapses had higher levels of IL-23 at presentation than patients without relapses $(p=0.021)$. Additionally, the levels of IL-23 were higher at the time of relapse compared to the last follow-up point before relapse. IL-23 might present a promising biomarker of uncontrolled and active disease and could give early indication of upcoming relapses.

Keywords: giant cell arteritis, glucocorticoids, leflunomide, follow-up, biomarkers, disease monitoring 


\section{INTRODUCTION}

Giant cell arteritis (GCA) is a granulomatous vasculitis affecting large- and medium-sized arteries (1). In the majority of patients, cranial and extracranial large arteries are involved to different degrees, leading to specific clinical phenotypes (2). Predominant cranial GCA (C-GCA) is characterized by headache, jaw claudication and visual disturbances, while clinical signs and symptoms of extra-cranial [large vessel (LVGCA)] typically include weight loss, myalgia and fever (3). Erythrocyte sedimentation rate (ESR) and/or levels of Creactive protein $(\mathrm{CRP})$ are usually increased at presentation in GCA patients, indicating a strong acute inflammatory response $(4,5)$.

High dose glucocorticoids (GC) represent the first-line treatment for GCA (6). They effectively control systemic inflammation and successfully prevent ischemic complications, such as acute vision loss. Relapses, however, are common when GC tapering regimen is applied, most likely due to ongoing inflammation in the affected vascular tissues, not adequately suppressed by GC (7). In addition, long-term use of GC is associated with various adverse effects, including bone fractures, infections, diabetes mellitus, and hypertension (8). Several other disease modifying anti-rheumatic drugs have subsequently been investigated for their steroid-sparing effect in GCA (9). So far, only tocilizumab showed the efficacy for achieving a sustained remission at week 52 of follow-up compared to placebo $(10,11)$. However, it was recently discovered by magnetic resonance angiography that signs of vascular inflammation persist in two-thirds of GCA patients treated with tocilizumab, despite clinical remission (12). Other agents, such as methotrexate exhibited limited or no evidence of benefit in the treatment of GCA (13). Leflunomide, on the other hand, has been shown to be effective and safe in reducing the rate of relapses in GCA in a small open-label study (14).

Classical acute phase parameters, such as ESR and CRP are commonly used for monitoring GCA activity $(15,16)$. However, measuring ESR and CRP to predict relapses has a limited value, since GC strongly suppress the systemic acute phase response, decrease ESR, as well as serum levels of CRP, despite an ongoing local vascular inflammation $(17,18)$. Subsequently, one cannot determine whether patients in GC-free remission are truly in remission or are still suffering from an ongoing subclinical disease. Therefore, new biomarkers are needed to predict the risk of relapses, and monitor disease activity in patients with GCA.

Van Sleen et al. (19) demonstrated higher numbers of monocytes and neutrophils, and lower numbers of natural killer (NK) and B cells in therapy-naïve GCA patients compared to healthy blood donors (HBDs). During GC treatment, as well as in GC-free remission, myeloid subsets remained elevated, while lymphoid subsets fluctuated substantially (19). Additionally, an altered phenotype of circulating neutrophils was also reported. The neutrophil phenotype changed from activated and highly adhesive, in the early stages of GCA, to a less adhesive after $48 \mathrm{~h}$ of GC treatment. However, 24 weeks following GC treatment and therapy tapering, neutrophils with the activated phenotype reappeared exhibiting a high expression of adhesion molecules L-selectin (CD62L) and integrin $\alpha \mathrm{M}$ (CD11b) (20). Long-term monitoring of the neutrophil phenotype could point to an incompletely controlled disease process (e.g., relapse) (21).

Previously, our cross-sectional study revealed significantly higher levels of serum amyloid A (SAA), interleukin (IL)-6, IL-8, IL-18, IL-23 and chitinase 3 like protein 1 (CHI3L1) in sera of therapy-naïve GCA patients compared to HBDs, reflecting an active disease (22). SAA has recently gained more attention in GCA (23), since it has been found to be highly elevated in GCA patients with active vs. inactive disease (24), and associated with relapses and visual disturbances (22).

In the current study, we longitudinally monitored the quantitative changes in leukocyte subtypes, neutrophil expression of adhesion molecules (CD62L, CD11b) and serum levels of selected analytes in GCA patients to evaluate the short- and long-term effects of GC vs. GC and leflunomide. Our aim was to identify candidate cellular and molecular biomarkers that could help monitoring disease activity and predicting the risk of a relapse.

\section{METHODS}

\section{Patients}

Thirty-one consecutive therapy-naïve GCA patients were enrolled in the study between October 2016 and October 2017. The diagnosis was established based on the 1990 ACR classification criteria (25) and a positive temporal artery biopsy (TAB) or positive color Doppler sonography (CDS) of temporal arteries. Blood samples from GCA patients were obtained at baseline visit [before initiation of GC therapy $\left(\mathrm{T}_{0}\right)$ ], as well as during follow-up at weeks 1, 4, 12, 24 and 48, unless otherwise stated. GC treatment was initiated at the time of diagnosis (Figure 1) in accordance with the unified protocol following the EULAR guidelines (26). Tapering of GC started at week 4 after baseline visit. Leflunomide (10 $\mathrm{mg}$ /day) was introduced as an adjuvant therapy at the week 12 to $17 / 31$ patients. $2 / 17$ patients experiencing adverse events (e.g., hair loss, diarrhea), discontinued leflunomide therapy and were consequently excluded from the longitudinal analysis. During follow-up and GC tapering, 4/31 patients experienced disease relapse after having already responded to GC therapy. Relapse was defined as the need for treatment intensification following new or increasing clinical symptoms typical of GCA. At the time of relapse, these patients were on GC monotherapy and consequently received leflunomide (10 mg/day), in addition to GC. From 3 relapsing patients, data was collected at the time before relapse (in remission), at the time closest to relapse (active disease) and 12 weeks after relapse (in remission). One patient relapsed in week 57 after diagnosis (after the last study follow-up point) and was excluded from the longitudinal analysis due to missing data (Figure 1). Patients and their samples were anonymized, before being used in the analyses. All patients signed informed consent to participate in the study. The study was approved by 
TIMELINE

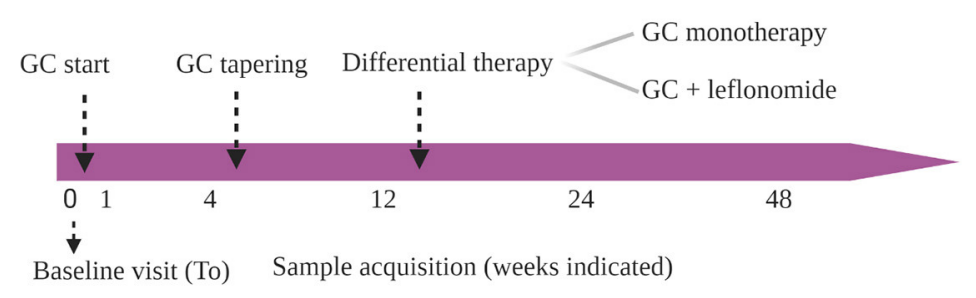

BASELINE VISIT

To

LONGITUDINAL ANALYSIS

Weeks 1, 4, 12

Weeks 24, 48

THERAPY-NAIVE GCA PATIENTS (To)

$$
\mathrm{N}=31
$$

Leukocyte subsets

Neutrophil CD62L and CD11b

Serum analytes

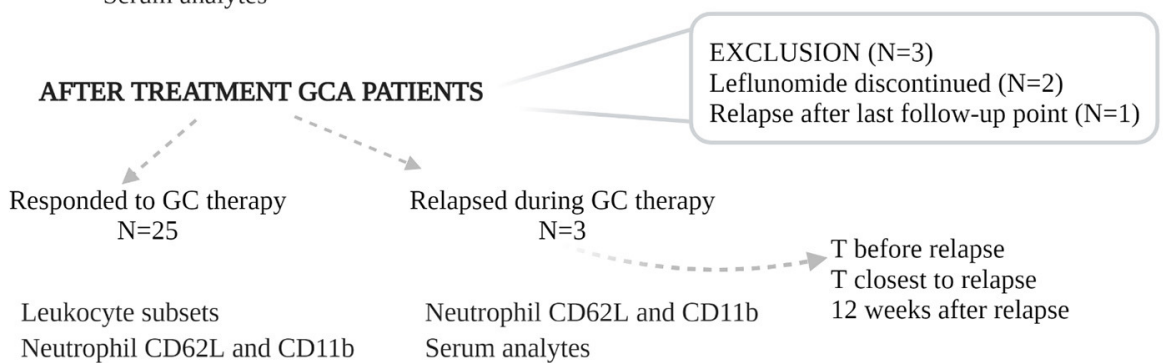

Serum analytes

\section{GC monotherapy $\mathrm{N}=10 / 25$}

Neutrophil CD62L and CD11b Serum analytes

\section{GC + leflunomide $\mathrm{N}=15 / 25$}

Neutrophil CD62L and CD11b

Serum analytes

FIGURE 1 | Overview of the study design. Thirty-one consecutive, therapy-naïve GCA patients were included in the study. Blood samples from GCA patients were obtained at baseline visit [before initiation of GC therapy (To)], and during follow-up at weeks 1, 4, 12, 24, and 48. GC treatment was initiated at the time of diagnosis. GC tapering started at week 4 after sampling. Leflunomide (10 mg/day) was introduced as an adjuvant therapy at week 12 to 17 GCA patients (two of them experienced therapy-related adverse events and were excluded from longitudinal analysis). During follow-up and GC tapering, three of 25 patients experienced disease relapse after they already responded to GC therapy. At the time of relapse these patients were on GC monotherapy and consequently received leflunomide (10 mg/day), in addition to GC. From 3 relapsing patients, the data was collected before relapse, at the time closest to relapse and 12 weeks after relapse. GC, glucocorticoids; GCA, giant cell arteritis; T, time.

the Slovenian National Medical Ethics Committee (\#99/04/15 and \#65/01/17).

\section{Histological Examination of Temporal Artery Biopsies and Routine Laboratory Parameters}

Histological analyses were performed on formalin-fixed paraffin-embedded sections stained with hematoxylin and eosin. Arterial wall inflammatory infiltrate and arterial occlusion were semiquantitatively scored. Arterial occlusion was considered when luminal stenosis was $>75 \%$.

Among laboratory parameters, ESR was measured by the WesternGreen method, CRP using Siemens Advia colorimetric assay, fibrinogen was detected by Siemens BCS XP/modified Clauss method, ferritin using the Advia chemiluminescence assay, SAA and haptoglobin were determined using immunonephelometry from Siemens (BN Prospec System and $\mathrm{BN}$ II, respectively).

\section{Flow Cytometry}

Venous blood was drawn from GCA patients into heparincontaining tubes. Whole blood immunophenotyping was performed using 7-Color Immunophenotyping kit with the following antibodies (Miltenyi Biotec, catalog \#130-098-456): CD14-FITC (clone Tük4), CD56-PE (clone REA196), CD16-PE (clone REA423), CD4-PerCP (clone VIT4), CD19-PE-Vio ${ }^{\circledR} 770$ (clone LT19), CD3-APC (clone BW264/56), CD8-APC-Vio 770 (clone BW135/80), CD45-VioBlue ${ }^{\circledR}$ (clone 5B1). Briefly, $100 \mu \mathrm{l}$ of whole blood was incubated with $10 \mu \mathrm{l}$ immunophenotyping reagent for $10 \mathrm{~min}$ in the dark, at $4^{\circ} \mathrm{C}$. After incubation, whole blood was lysed using Red Blood Lysing Solution (Miltenyi Biotec, catalog \#130-098-456). Neutrophil phenotyping was performed in $50 \mu \mathrm{l}$ of whole blood, incubated for $30 \mathrm{~min}$ at $4^{\circ} \mathrm{C}$ in the dark, with the following antibodies (eBioscience): CD16-PE (clone eBioCB16; catalog \#50-112-4738), CD62LPE-Cy5 (clone DREG56; catalog \#50-140-71) and CD11b-APC (clone ICRF44; catalog \#17-0118-42). After incubation, samples 
were lysed, using Whole Blood Lysing Reagent Kit (Beckman Coulter; catalog \#6602764). All samples were analyzed using flow cytometer MACSQuant Analyzer 10 (Miltenyi Biotec). Analysis of flow cytometry data was performed using MACSQuantify (Analysis Software version 2.8, Miltenyi Biotec) and FlowLogic (Flow Cytometry Analysis Package, version 7.00.0a, Invasion Software Technologies Pvt Ltd).

\section{Biomarker Protein Detection}

Serum concentrations of IL-8, IL-18, IL-23, CHI3L1 and soluble CD62L (sCD62L) were measured by MagPix (Luminex xMAP Technology) using human pre-mixed multi-analyte kits (R\&D Systems; catalog \#LXSAHM) and IL-6 using ELISA (Invitrogen; catalog \#KHC0061).

\section{Statistical Analysis}

Statistical analysis was performed using SPSS statistical software package version 22.0 and Graph Pad Prism software 9.0. The normality of data distribution was investigated by the Shapiro-Wilk test. Due to the non-normal distribution of the data, summary statistics are expressed as medians and 2575th percentiles $\left(\mathrm{Q}_{25}-\mathrm{Q}_{75}\right)$. Mann-Whitney U-test was used to compare medians of measured parameters in GCA patients with or without specific clinical signs/symptoms. Statistical analysis of longitudinal data was performed using Kruskal-Wallis test followed by Dunn's multiple comparison test, which calculates adjusted $p$-values. All tests were two-tailed and $p$-values of $<0.05$ were regarded as statistically significant.

\section{RESULTS}

\section{Baseline Visit}

The median $\left(\mathrm{Q}_{25}-\mathrm{Q}_{75}\right)$ age of the included patients was 74.9 (68.0-76.8) and there were 20 (65\%) females. The most frequent clinical symptoms/signs reported were newly formed headache (74\%), jaw claudication (65\%) and general symptoms (71\%). Visual disturbances were present in eight (26\%) patients and seven patients $(23 \%)$ had LV-GCA. The median $\left(\mathrm{Q}_{25}-\mathrm{Q}_{75}\right)$ ESR was $78.0(48.0-94.5) \mathrm{mm} / \mathrm{h}$ and the median CRP value was 71.5 (34.3-128.8) mg/l (Table 1).

\section{Therapy-Naïve GCA Patients With Transmural Inflammation and Occlusion of Temporal Arteries Have Higher Serum Levels of CHI3L1}

To reveal if the inflammatory process in TABs of therapy-naïve GCA patients associates with the numbers of leukocyte subsets and serum parameters, we correlated the measured baseline cell and serum parameters with the presence of histological signs of GCA (transmural inflammation, occlusion of temporal arteries), clinical symptoms and signs and development of a future relapse.

Histological examination of TAB was performed in 23 therapy-naïve GCA patients. Signs of transmural inflammation $(\mathrm{TAB}+\mathrm{GCA})$ and vessel occlusion were found in 18 (78\%) and 8 (26\%) of the examined TABs, respectively (Table 1). In general, the patients with transmural inflammation had significantly higher ESR $(p=0.0443)$, haptoglobin $(p=0.0470)$ and CHI3L1 $(p=0.0279)$ compared to patients with no signs of inflammation
TABLE 1 | Demographics, clinical and laboratory data of therapy-naïve GCA patients at baseline visit.

\begin{tabular}{|c|c|}
\hline \multicolumn{2}{|l|}{ Demographic data } \\
\hline Number of patients & 31 \\
\hline Median age in years $\left(Q_{25}-Q_{75}\right)$ & $74.9(68.0-76.8)$ \\
\hline Number of females (\%) & $20(65)$ \\
\hline Median duration of symptoms (days) $\left(Q_{25}-Q_{75}\right)$ & $30(30-60)$ \\
\hline Median body mass index $\left(\mathrm{kg} / \mathrm{m}^{2}\right)\left(\mathrm{Q}_{25}-\mathrm{Q}_{75}\right)$ & $23.8(21.3-28.7)$ \\
\hline \multicolumn{2}{|l|}{ Symptoms and signs $n(\%)$} \\
\hline General symptoms & $22(71)$ \\
\hline Fever & $5(16)$ \\
\hline Weight loss & $19(61)$ \\
\hline Headache & $23(74)$ \\
\hline Jaw claudication & $20(65)$ \\
\hline Scalp tenderness & $12(39)$ \\
\hline Visual disturbances & $8(26)$ \\
\hline Dry cough & $5(16)$ \\
\hline Large vessel involvement & $7(23)$ \\
\hline \multicolumn{2}{|l|}{ Histological examination of TABs $n(\%)$} \\
\hline TAB performed & $23(74)$ \\
\hline Transmural inflammation & $18(78)$ \\
\hline Vessel occlusion & $8(34)$ \\
\hline \multicolumn{2}{|c|}{ Ultrasound examination of temporal arteries $n(\%)$} \\
\hline HALO effect & $28(90)$ \\
\hline \multicolumn{2}{|l|}{ Median laboratory values $\left(Q_{25}-Q_{75}\right)$} \\
\hline $\operatorname{ESR}(\mathrm{mm} / \mathrm{h})$ & $78.0(48.0-94.5)$ \\
\hline CRP (mg/l) & 71.5 (34.3-128.8) \\
\hline Fibrinogen (g/l) & $6.2(5.7-7.2)$ \\
\hline Ferritin (g/l) & $258(161-441)$ \\
\hline Haptoglobin (g/l) & $4.5(2.6-5.6)$ \\
\hline
\end{tabular}

CRP, C-reactive protein; ESR, erythrocyte sedimentation rate; GCA, giant cell arteritis; $T A B$, temporal artery biopsy.

in the TABs (TAB- GCA) (Figure 2A). GCA patients with occlusion of the temporal arteries had higher amount of CHI3L1 $(p=0.0306)$ but lower neutrophil expression of CD11b ( $p=$ $0.0017)$ compared to GCA patients without vessel occlusion (Figure 2B). Other measured parameters (serum analytes, the number of leukocyte subsets, the expression of CD62L) did not associate with temporal artery transmural inflammation or occlusion.

Correlating the clinical signs and symptoms with the measured parameters, we found that therapy-naïve GCA patients with visual disturbances $(n=8)$ had lower median amount of serum IL-23 compared to patients without visual disturbances ( $n$ $=23 ; p=0.047$, Figure 2C). Additionally, the median amount of IL-23 was significantly higher in the serum of therapy-naïve GCA patients (at baseline visit) who developed a future relapse $(n=4)$ compared to patients with no future relapses $(n=27 ; p=0.021)$ (Figure 2D). Other clinical symptoms and signs at baseline visit did not correlate with measured cell and serum parameters.

\section{Longitudinal Follow-Up}

The longitudinal analysis included 28 of the initial 31 GCA patients, who received GC immediately after pre-treatment 


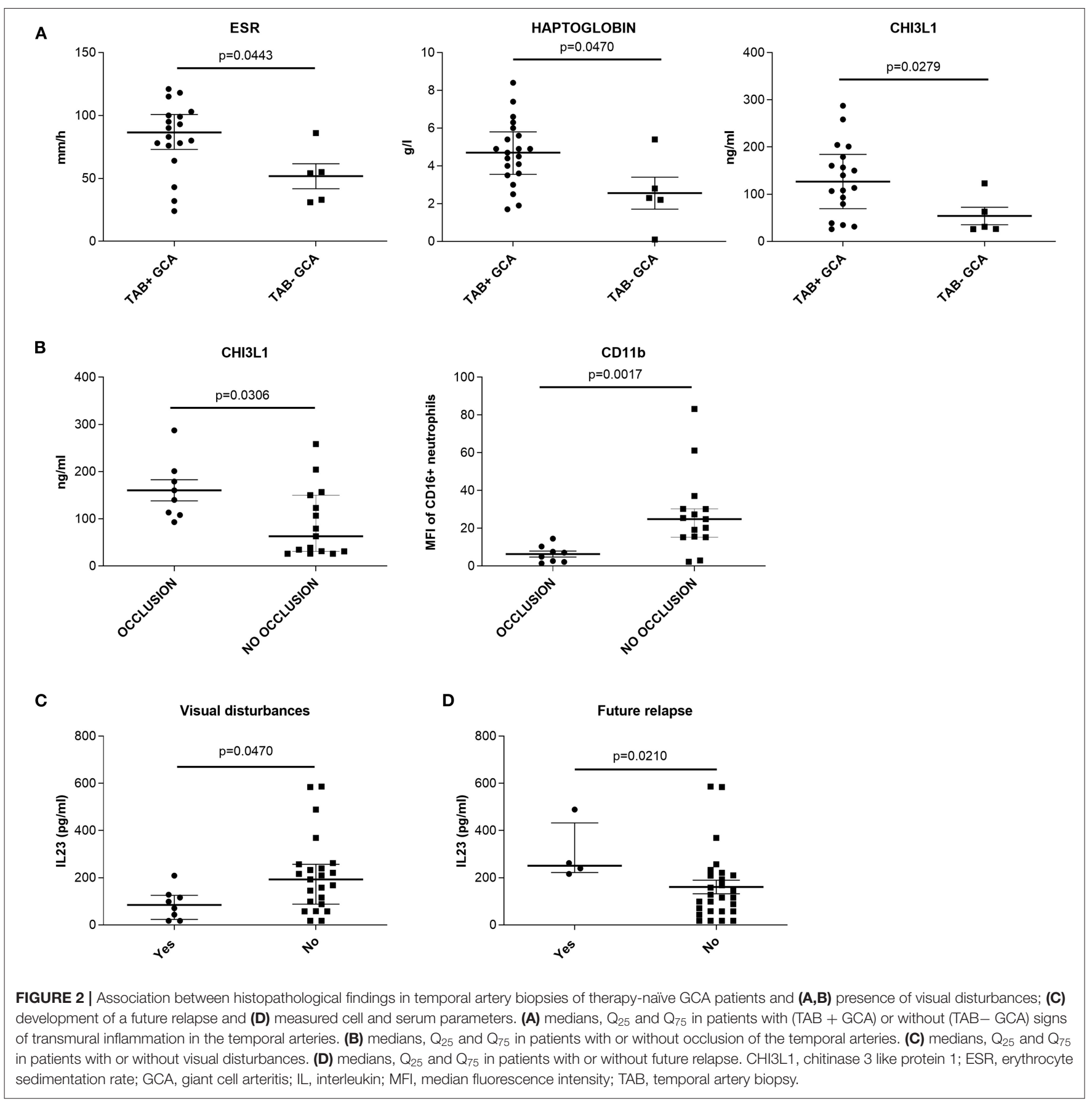

(baseline) sampling. Among them, 25 responded to GC at the initial dosage, with prompt improvement of clinical signs and symptoms of GCA, as determined by a rheumatologist during the follow-up visits. These patients were able to continue GC reduction without any deviation from the protocol. Three patients (9.7\%) experienced a relapse following a period of remission during the 48 weeks of follow-up. Patient 1 (P1) experienced a relapse at week 24 , P2 relapsed at week 48 and P3 relapsed at week 12. All relapsing patients were on GC monotherapy at the time of relapse. P1 and P2 received
GC dosage of $4 \mathrm{mg} /$ day, while $\mathrm{P} 3$ received $12 \mathrm{mg} /$ day. All three relapses were characterized by new or intensified clinical symptoms considered typical of GCA. P1 and P2 experienced signs of systemic inflammation (fever, weight loss, fatigue, myalgia), while P3 experienced cranial signs (headache, jaw claudication). P1 and P2, but not P3, also had increased ESR and CRP, associated with GCA in the absence of an alternative explanation, compared to the last time point before relapse when they were in remission. All relapsing patients responded to additional therapy with leflunomide 
(10 mg/day), and were able to continue with GC tapering as scheduled.

\section{Longitudinal Analysis Shows Fluctuation in Leukocyte Subsets From Active (Pre-treatment) to Non-active (After Treatment) GCA}

To get insight into the effects of GC treatment on alterations of leukocyte subset composition during GC therapy, we obtained longitudinal profiling data for immune cells at $\mathrm{T}_{0}$ and 1,4 , and 12 weeks of follow up for 16 GCA patients. These patients were on GC monotherapy and responded to GC treatment.
At week 1 after GC treatment, the number of circulating CD4+ T-lymphocytes $(p=0.019)$ and B-lymphocytes $(p=$ 0.002 ) significantly increased in GCA patients compared to $\mathrm{T}_{0}$. The number of CD4+ T-lymphocytes then diminished, reaching the pre-treatment $\left(\mathrm{T}_{0}\right)$ numbers at weeks $4(p=$ 0.0009 vs. week 1$)$ and 12 ( $p=0.044$ vs. week 1$)$, while the number of B-lymphocytes only slightly decreased. In contrast, the number of neutrophils progressively increased over the weeks 1 and 4 and was significantly elevated at week 12 compared to $\mathrm{T}_{0}(p=0.0003)$. No significant differences were observed in the number of monocytes,
A

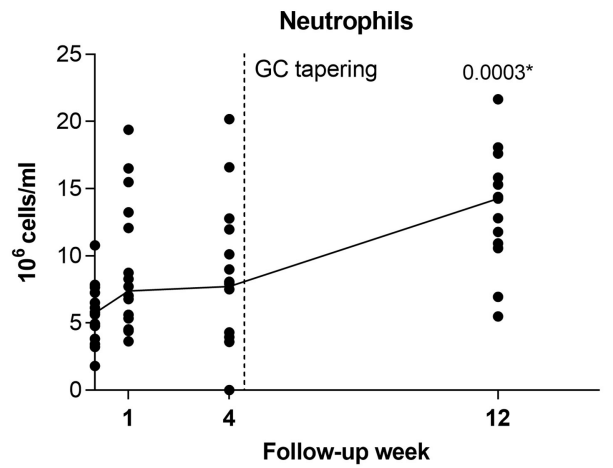

C

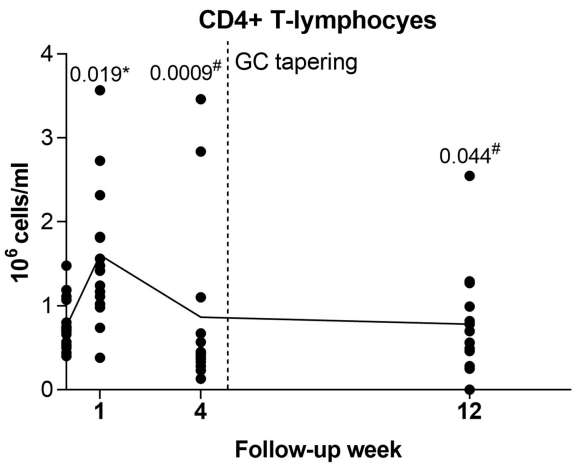

E

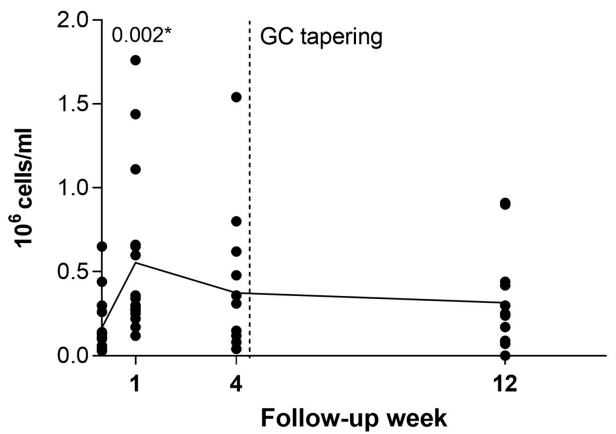

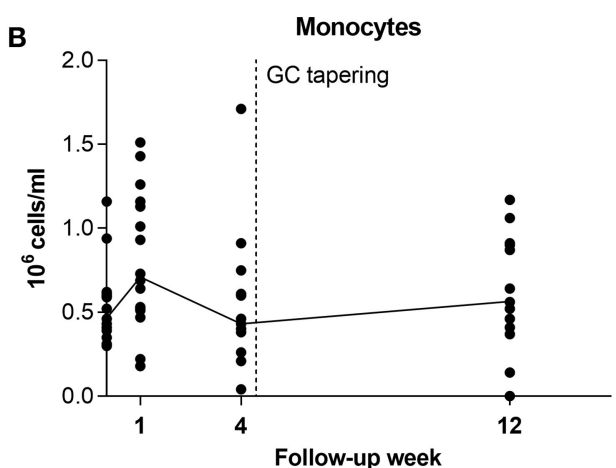

D
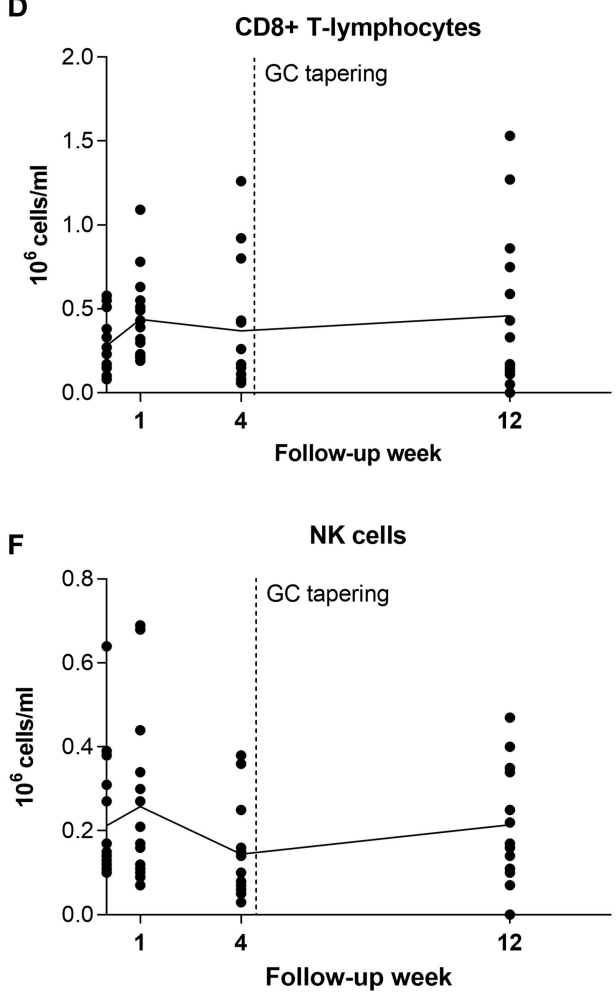

FIGURE 3 | Longitudinal analysis of leukocyte subsets in GCA patients before and during therapy with GC. After pre-treatment sampling, all patients received GC and therapy tapering started after week 4 (indicated with vertical dotted line). Shown are medians from each follow-up time point (black horizontal connecting line) for (A) neutrophils; (B) monocytes; (C) CD4 + T-lymphocytes; (D) CD8+ T-lymphocytes; (E) B-lymphocytes; and (F) NK cells. GC, glucocorticoids; GCA, giant cell arteritis; NK, natural killer. * indicates statistical significance between the corresponding timepoint and $\mathrm{T}_{0}$; ${ }^{\#}$ indicates statistical significance between the corresponding timepoint and week 1. 
CD8+ T-lymphocytes and NK cells during the 12 weeks of follow-up (Figure 3).

\section{Neutrophil Adhesion Molecules Are Differentially Expressed in Active Compared to Non-active GCA}

To confirm the effect of GC and leflunomide on neutrophil phenotype, we measured neutrophil CD62L and CD11b expression in peripheral blood of 25 GCA patients who responded to therapy, at $\mathrm{T}_{0}$ and at weeks $1,4,12,24$, and 48 of follow-up.

No significant differences in the neutrophil CD62L and CD11b expression were observed at $\mathrm{T}_{0}$ or at week 12, prior to leflunomide addition, between patients receiving GC and patients who later received GC and leflunomide (Supplementary Table 1). The median expression of CD62L on neutrophils, decreased in GCA patients, responding to therapy, from $\mathrm{T}_{0}$ to weeks 1,4 , and 12 after GC treatment (Figure 4A). At week 48, there was a distinct elevation in CD62L: patients receiving GC only, showed a marked increase, compared to $\mathrm{T}_{0}$, while in patients additionally receiving leflunomide, the CD62L expression was consistently low. However, the difference between the two groups was not significant. The median neutrophil expression of $\mathrm{CD} 11 \mathrm{~b}$ declined from $\mathrm{T}_{0}$ to week 4 , but increased and reached median pre-treatment expression levels at week 12. Patients receiving GC only, showed an increase in CD11b expression at week 24 compared to patients receiving leflunomide in addition to GC, however both treatment groups exhibited similar CD11b expression at week 48 (Figure 4A). The differences in CD11b expression between different time points or differential therapy groups were not statistically significant.

Since the activation of neutrophils causes CD62L shedding from the membrane into the bloodstream (23), we also measured serum levels of sCD62L at baseline and during the follow-up of GCA patients. In contrast to the changing expression of neutrophil CD62L, median levels of sCD62L remained constant during the entire follow-up time (Figure 4B). No correlation between neutrophil CD62L and $s$ CD62L was determined at $\mathrm{T}_{0}$ or any of the follow-up points (Supplementary Figure 1).

\section{Serum Biomarker Levels Decrease From Active to Non-active GCA Depending on the Type of Treatment}

To identify serum biomarkers that can be used to monitor GCA activity and could inform on the ongoing vascular inflammation, we determined serum levels of a predefined set of proteins (SAA, IL-6, IL-8, IL-18, IL-23, CHI3L1) in 25 GCA patients who responded to therapy at $\mathrm{T}_{0}$ and at weeks $4,12,24$, and 48 of follow-up.

No significant differences in measured analytes were observed at $\mathrm{T}_{0}$ or at week 12, prior to leflunomide addition, between patients receiving GC and patients who later received GC and leflunomide (Supplementary Table 1). Importantly, the median levels of SAA $(p=0.0099)$ and IL-6 $(p=0.0001)$ decreased significantly at week 4 after GC treatment compared to $\mathrm{T}_{0}$. Both analytes remained low at weeks 12,24 , and 48 compared to $T_{0}$ in both groups of patients although significance was only reached for patients under combinatorial therapy with leflunomide $(p$ $=0.0001$ for SAA at week $48 ; p=0.0012$ and $p=0.0054$ for
IL-6 at weeks 24 and 48, respectively). Median levels of serum IL-8, IL-18 and CHI3L1 remained stable during the entire followup, regardless of therapy used. Median IL-23 decreased from $\mathrm{T}_{0}$ to week 48 in patients receiving GC monotherapy, while in patients under combinatorial therapy there was an increase at weeks 24 and 48 compared to week 12, although this was not significant (Figure 4B).

\section{IL-23 Is Increased in Relapsing Patients}

The expression of neutrophil CD62L and CD11b, as well as the levels of sCD62L in the group of relapsing patients varied greatly between the three patients (Figures 5A,B). This variation might also be attributed to the different time points when the patients relapsed and different GC dosages.

Acute phase reactants IL-6 and SAA showed different fluctuations in relapsing patients (Figure 5B). At the time of relapse (active disease) strong increase of IL-6 as compared to the last follow-up point before relapse (inactive disease) was observed for P1 and P2, while the increase in P3 who relapsed early in the course of the disease (week 12), while still on a high GC dose ( $48 \mathrm{mg} /$ day) was very low. A small increase in the level of SAA was observed in just one patient at the time of relapse, compared to the time point before relapse (Figure 5B). Since elevated levels of IL- 6 at the time of relapse could indicate the reactivation of GCA, we next determined how many patients in the responder group had elevated levels of IL-6 without disease flare during the follow-up period. We defined the number of patients with elevated levels of IL- 6 on two consecutive visits at weeks 12, 24 or 48 [similar to Stone et al. (17)] compared to the week 4 when patients were on high GC dose $(48 \mathrm{mg} /$ day) for the longest period of time (4 weeks). 15/25 (60\%) patients from the responder group (10/10 from GC only group and 6/15 from GC plus leflunomide group) exhibited elevated levels of IL-6 (at two consecutive visits at weeks 12,24 or 48 vs. week 4 ), despite disease inactivity and no subsequent relapse. We therefore next looked for other biomarkers that were elevated in all three relapsing patients with active (at the time of relapse) compared to nonactive (before relapse, in remission) disease. We identified three serum markers which met our criteria: IL-18, IL-23 and CHI3L1 (Figure 5B). Subsequently, we analyzed how many patients from the responder group had elevated levels of the three identified parameters during the follow-up without a subsequent relapse (the same as previously described for IL-6). 13 out of $25(52 \%)$ patients in the responder group had elevated IL-18, 9/25 (36\%) patients had elevated CHI3L1 and 6/25 (24\%) had elevated IL23 during the course of the disease in the absence of clinical manifestations indicating a relapse.

\section{DISCUSSION}

The current longitudinal study provides data on the effects of short- and long-term use of GC or GC, in combination with leflunomide on leukocyte subtype dynamics, neutrophil phenotype and serum analytes in GCA patients. The steroidsparing effect of leflunomide in GCA has been shown in an open-label study by Hočevar et al. (14). During the first 48 weeks of follow-up, $13.3 \%$ of GCA patients who received GC 
A

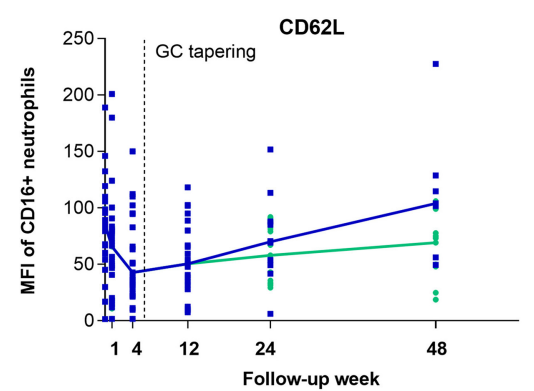

B

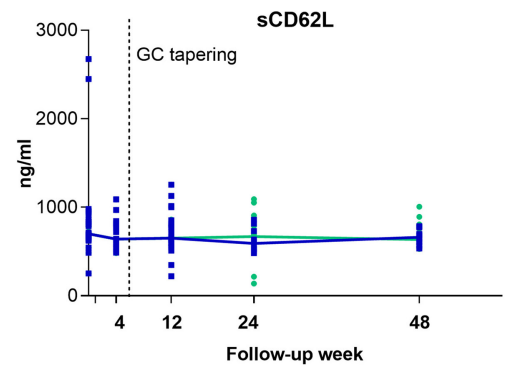

SAA

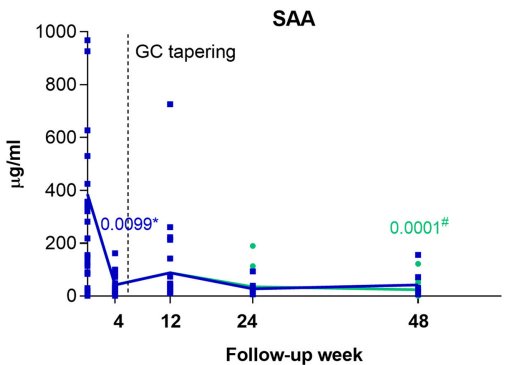

IL-8
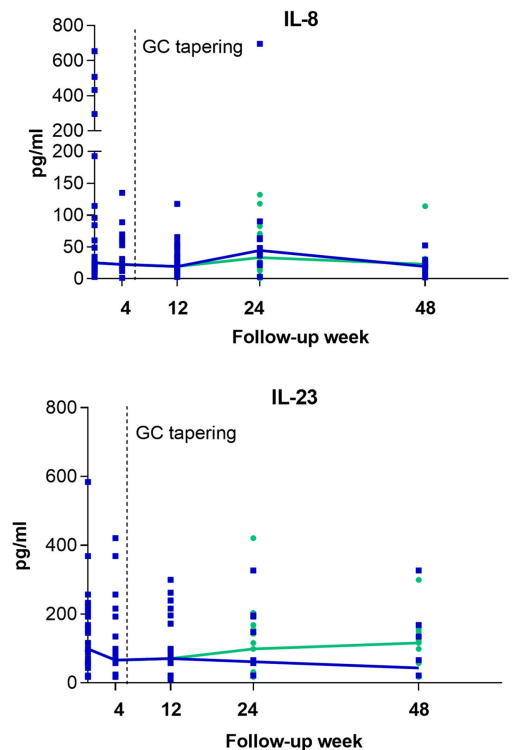

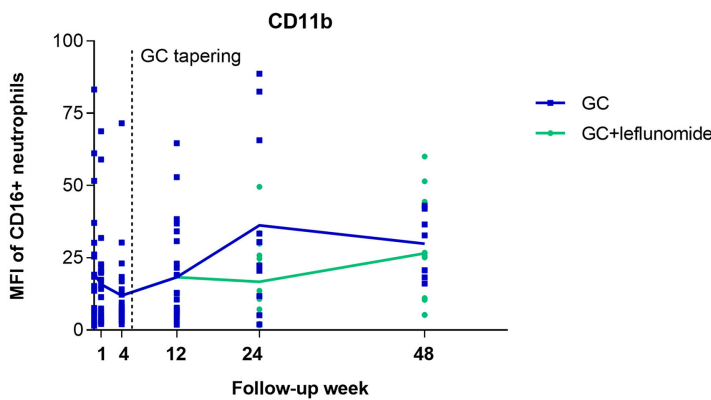

$\rightarrow G C$

$\rightarrow$ GC+leflunomide
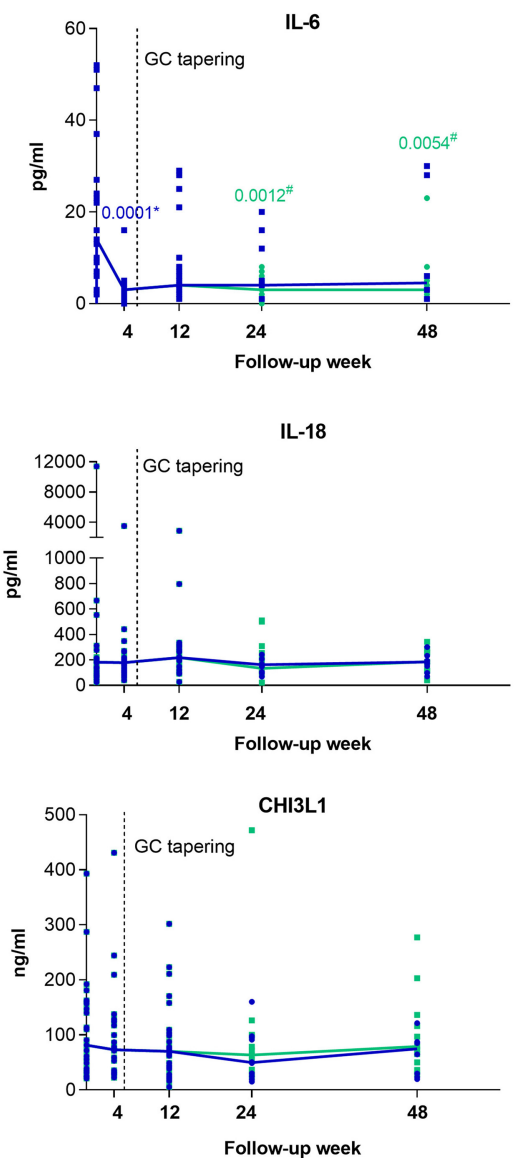

FIGURE 4 | Surface expression of CD62L and CD11b on neutrophils (A) and serum levels of selected analytes (B) from GCA patients who responded to therapy $(n=25)$, at baseline visit (To) and 1, 4, 12, 24, and 48 weeks of follow-up. After pre-treatment sampling (time point 0), all patients received GC and therapy tapering started after week 4 (indicated with vertical dotted line). After week 12 some of the patients (15/25) received leflunomide, in addition to GC therapy (green). The horizontal lines connect the medians from each follow-up time point. GCA, giant cell arteritis; MFI; median fluorescence intensity. ${ }^{*}$ indicates statistical significance between the corresponding timepoint and $\mathrm{T}_{0}$ in patients receiving GC monotherapy (blue); \# indicates statistical significance between the corresponding timepoint and $T_{0}$ in patients receiving $G C$ in combination with leflunomide (green). 
A

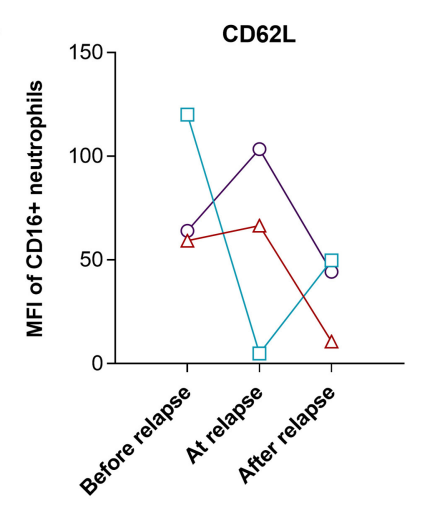

в
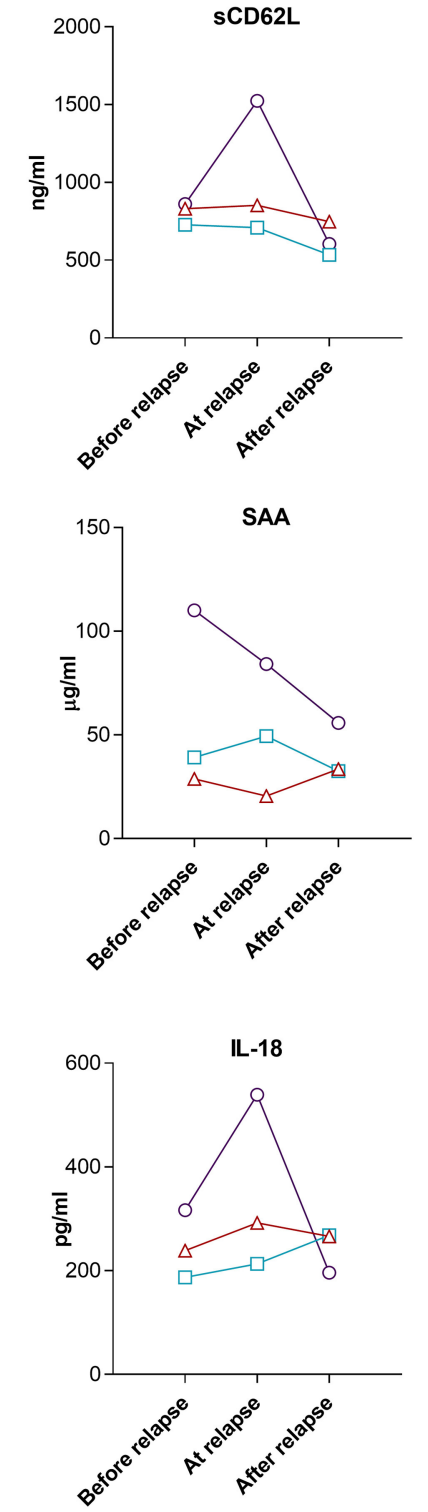

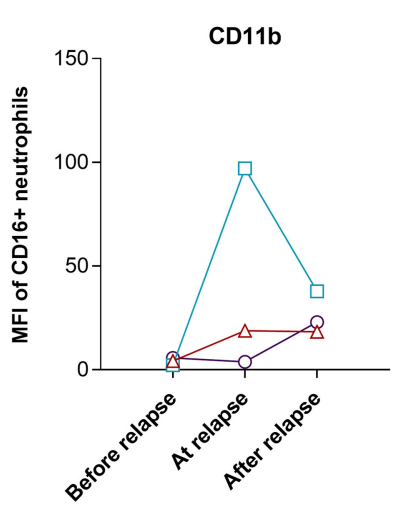

$-\mathrm{O}$ - P1

$-\square-P 2$

$\triangle \mathrm{P} 3$

$$
\begin{aligned}
& \multimap \text { P1 } \\
& -\square \text { P2 } \\
& -\triangle P 3
\end{aligned}
$$
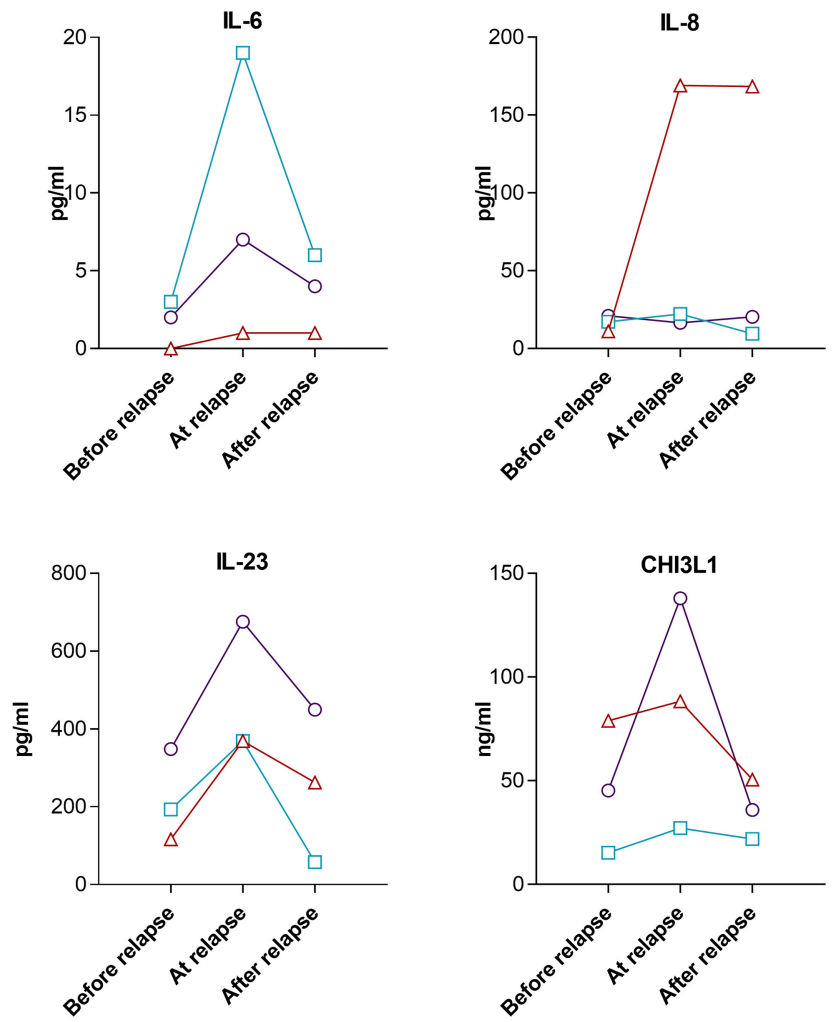

FIGURE 5 | Surface expression of CD62L and CD11b on neutrophils (A) and serum levels of selected analytes (B) from relapsing GCA patients during the 48 weeks of follow-up. Shown are levels/MFI at the last follow-up time point before relapse, at the time point closest to relapse and 12 weeks after relapse. P1 relapsed at week 24, P2 relapsed at week 48 and P3 relapsed at week 12. MFI, median fluorescence intensity, P, patient. 
in combination with leflunomide relapsed compared to $39.1 \%$ relapsing patients receiving GC monotherapy (14). We observed similar findings in the present study, where all three relapsing patients were receiving GC monotherapy and there were no relapses observed in patients under combinatorial therapy with leflunomide during the 48 weeks of follow-up. Furthermore, the relapsing patients received leflunomide at the time of relapse in addition to GC and they all entered into remission 12 weeks after relapse. Subsequently, they were able to adhere to GC tapering as initially scheduled.

We observed an immediate short-term effect of high dose $\mathrm{GC}$ on increasing the numbers of $\mathrm{CD}^{+}{ }^{+} \mathrm{T}$ - and $\mathrm{B}$ lymphocytes after 1 week, while long-term GC treatment (12 weeks) resulted in decreased numbers of $\mathrm{CD} 4+\mathrm{T}$ - and $\mathrm{B}$ lymphocytes, with increased numbers of neutrophils compared to the pre-treatment time. Similar findings were reported previously by other studies $(19,27)$. Van der Geest et al. observed the same increase in B cell count 2 weeks after GC treatment, however did not find any evidence of either $\mathrm{B}$ cell replenishment from the bone marrow or compensatory hyperproliferation of circulating $\mathrm{B}$ cells (27). An increase in B cell counts as a result of early GC treatment might reflect a redistribution or intravascular marginalization of $\mathrm{B}$ cells during active disease. Neutrophilia caused by GC is a known effect resulting from increased polymorphonuclear cell release from the bone marrow to the circulation and their increased survival. On the other hand, GC can reduce the migration of neutrophils into inflammatory sites by decreasing the surface expression of CD62L, thus preventing the adhesion of neutrophils and their tissue accumulation (28). The same was observed in the present study, in which the expression of CD62L rapidly decreased after initiation of GC treatment and began to increase progressively to week 48 , when patients received lower doses of GC monotherapy. The expression of CD62L was higher at week 48 compared to the pre-treatment timepoint. The activated $\mathrm{CD} 62 \mathrm{~L}^{\text {hi }} \mathrm{CD} 11 \mathrm{~b}^{\text {hi }}$ neutrophil profile in GCA patients at baseline has been reported in a previous study (20). Within 1 week of GC treatment, this phenotype was brought under control, as demonstrated by switching to $\mathrm{CD} 62 \mathrm{~L}^{\text {lo }} \mathrm{CD} 11 \mathrm{~b}^{\text {lo }}$ phenotype with reduced endothelial adhesion. However, 24 weeks after initiation of GC therapy and tapering, an escaped pro-inflammatory phenotype $\left(C D 62 L^{\text {hi }} C D 11 b^{\text {hi }}\right)$, with elevated endothelial adhesion was reported (20). The progressive increase of CD62L was not observed in our study in patients under combinatorial therapy with leflunomide. In contrast, the expression of CD62L decreased in these patients compared to the pre-treatment time. Leflunomide is a selective inhibitor of de novo pyrimidine synthesis (limiting proliferation of lymphocytes) and lowers the production of IL- 6 , TNF- $\alpha$, IL12 and IL-17. Moreover, leflunomide can affect the expression of adhesion molecules and reduces leukocyte adhesion to endothelial cells (29), which could explain the downregulation of CD62L neutrophil expression observed in our study. The addition of leflunomide might have a beneficial long-term effect on the control of neutrophil adhesion. However, no significant differences in the expression of CD62L were observed between relapsing patients and responders.
We and others have previously already determined higher serum levels of CHI3L1 in therapy-naive GCA patients compared to HBDs $(22,30)$, while in the present study, we additionally found that the levels of CHI3L1 were associated with signs of transmural inflammation and vessel occlusion in temporal arteries. The source of CHI3L1 in serum of GCA patients might stem from monocytes, macrophages and giant cells (30). Additionally, CHI3L1 is highly expressed in TABs of GCA patients, predominantly in the intima-media border region (30, 31). CHI3L1 is involved in tissue remodeling and angiogenesis (32), and deregulation of these processes in GCA TABs might contribute to increased amounts of CHI3L1 in occluded temporal arteries. Our finding suggests that $\mathrm{CHI} 3 \mathrm{~L} 1$ is mainly released in fully developed GCA with transmural inflammation and lumen occlusion. Although in vitro production of CHI3L1 by macrophages has been shown to be sensitive to GC (33), we observed only a slight reduction in serum CHI3L1 levels between baseline and follow-up visits, indicating that cells producing CHI3L1 may be GC resistant. In line with these observations, GCA patients with extensive transmural inflammation and remodeling of temporal arteries had higher levels of CHI3L1 that might require a therapeutic approach different from the currently established GC. Targeting CHI3L1 in GCA may inhibit macrophages that might currently be insufficiently suppressed by GC (34).

Traditional inflammatory parameters, such as ESR and CRP have been described as insufficient markers for monitoring disease activity in GCA (17). In the current study, increased ESR and CRP were observed in only two out of three relapsing patients at the time of relapse when the disease clinically reactivated. Tocilizumab additionally suppresses these markers $(17,35)$, indicating the need for new inflammatory markers to aid in monitoring GCA activity during treatment. Our results demonstrated the suppressive effect of GC on systemic levels of additional acute phase reactants, such as SAA and IL-6 which limits their use as markers of disease activity during therapy. Similar to our results, Dartevel et al. showed significantly higher SAA levels in patients with active (newly diagnosed and relapsing) compared to inactive GCA (responding to therapy). However, the authors did not compare the difference in SAA levels in relapsing patients at the time before, at and after relapse (24). In contrast to SAA, levels of IL-6 increased in all three relapsing patients, in our study, when the disease reactivated, but also in $60 \%$ of the responder patients at two consecutive visits at weeks 12,24 or 48 compared to week 4 . All 10 patients in the responder group that received GC monotherapy had increased levels of IL-6, while this was observed in only 5/15 patients under combinatorial therapy with leflunomide. Since increasing the doses of GC to completely suppress serum IL-6 would lead to the higher rate of treatment related adverse effects, the addition of leflunomide could be a better option. As seen in the present study, leflunomide may have a beneficial effect on reducing both systemic and vascular inflammation. CHI3L1, on the other hand, was increased in all three relapsing patients at the time of relapse and was elevated in only $36 \%$ of the patients who responded to therapy with GC or GC and leflunomide. This might indicate an incompletely controlled disease process in these patients that may 
lead to a future relapse, but needs to be confirmed on a larger cohort of patients after a longer follow-up period.

Prior to treatment, relapsing patients had significantly elevated levels of IL-23 compared to patients without relapses. The levels of IL23 were also higher in all three relapsing patients, at the time closest to relapse, compared to the last time point before relapse. Levels of IL-23 decreased again after patients entered into remission and concurrently received leflunomide. Conway et al. similarly found significantly increased expression of IL-23 in the TABs of GCA patients with two or more relapses compared to patients without or with only one relapse (36). IL-23 is pivotal in differentiation of Th17 cells, producing IL-17A with pleiotropic effects on a variety of cells, including macrophages, neutrophils, endothelial cells and fibroblasts, and actively contributes to inflammatory cascades (37). IL-23 seems to be GC-dependent, since in our study, its levels decreased substantially from baseline visit to week 48 of follow-up in patients treated with GC monotherapy, however it remained elevated in GCA patients who experienced a relapse. Higher levels of IL-23 might indicate an ongoing vascular tissue inflammation in relapsing patients, inferring that IL-23 might serve as a marker of persistent, active disease and as a relapse predictor in GCA patients. Although patients receiving GC and leflunomide had slightly higher levels of IL-23 during the 48 weeks follow-up period, none of them developed a relapse. This might be associated with the primary effect of leflunomide inhibiting T-lymphocyte proliferation $(29,38)$ and thus inhibiting the IL-23-driven polarization toward the Th17 lineage $(37,39)$. As IL23 is most strongly expressed and produced by macrophages and dendritic cells $(40,41)$, this (in addition to CHI3L1) might represent another clue for potential therapeutic benefits of suppressing macrophage activation in GCA.

The strengths of our current report are the prospective study design, and the uniform clinical evaluation, with known dates of GC therapy start and tapering at follow-ups. Moreover, GCA patients joined our study when they were therapy-naive, which allowed us to evaluate the effects of active disease. Previous longitudinal studies often included patients already treated with GC.

The major limitation of our study is a relatively small number of included and longitudinally followed GCA patients $(n=25)$, as well as the small number of relapsing patients $(n=3)$ that explains the lack of statistical significance. The patients were only followed up to 48 weeks when they did not yet achieve

\section{REFERENCES}

1. Jennette JC, Falk RJ, Bacon PA, Basu N, Cid MC, Ferrario F, et al. 2012 revised international chapel hill consensus conference nomenclature of vasculitides. Arthritis Rheum. (2013) 65:1-11. doi: 10.1002/art.37715

2. Camellino D, Matteson EL, Buttgereit F, Dejaco C. Monitoring and longterm management of giant cell arteritis and polymyalgia rheumatica. Nat Rev Rheumatol. (2020) 16:481-95. doi: 10.1038/s41584-020-0458-5

3. Koster MJ, Matteson EL, Warrington KJ. Large-vessel giant cell arteritis: diagnosis, monitoring and management. Rheumatology. (2018) 57(Suppl. 2):ii32-42. doi: 10.1093/rheumatology/kex424
GC-free remission and we only assessed their peripheral blood that may not completely reflect the pathological processes at the sites of tissue inflammation. Future longitudinal studies with similar designs could provide further insights by increasing the number of patients, tested parameters and follow-up time, as well as assessing the vascular pathological processes in the temporal arteries in greater detail.

\section{DATA AVAILABILITY STATEMENT}

The raw data supporting the conclusions of this article will be made available by the authors, without undue reservation.

\section{ETHICS STATEMENT}

The studies involving human participants were reviewed and approved by National Medical Ethics Committee of Slovenia. The patients/participants provided their written informed consent to participate in this study.

\section{AUTHOR CONTRIBUTIONS}

TK, KL, SS-Š, and AH designed the experiments. TK, KL, and $\mathrm{PŽ}$ performed the flow cytometry analysis and conducted the serum biomarker experiments. AK supported the analysis of flow cytometry study. TK and GT performed the statistical and bioinformatic analyses. $\mathrm{AH}$ and MT conducted the clinical evaluation of the patients. SS-Š, SČ, AH, and MT coordinated the study. MF-B provided critical input to data analysis, visualization and interpretation. TK wrote the original draft. All authors reviewed, edited the final draft, authors have seen, approve the manuscript and its contents, and as well as are aware of the responsibilities connected with the authorship.

\section{FUNDING}

This work was supported by the Slovenian Research Agency ARRS with funding grant \#P3-0314.

\section{SUPPLEMENTARY MATERIAL}

The Supplementary Material for this article can be found online at: https://www.frontiersin.org/articles/10.3389/fmed. 2021.827095/full\#supplementary-material
4. Chan FLY, Lester S, Whittle SL, Hill CL. The utility of ESR, CRP and platelets in the diagnosis of GCA. BMC Rheumatol. (2019) 3:14. doi: 10.1186/s41927-019-0061-z

5. Kermani TA, Schmidt J, Crowson CS, Ytterberg SR, Hunder GG, Matteson $\mathrm{EL}$, et al. Utility of erythrocyte sedimentation rate and C-reactive protein for the diagnosis of giant cell arteritis. Semin Arthritis Rheum. (2012) 41:86671. doi: 10.1016/j.semarthrit.2011.10.005

6. Hellmich B, Agueda A, Monti S, Buttgereit F, de Boysson H, Brouwer E, et al. 2018 Update of the EULAR recommendations for the management of large vessel vasculitis. Ann Rheum Dis. (2020) 79:19-30. doi: 10.1136/annrheumdis-2019-215672 
7. Maleszewski JJ, Younge BR, Fritzlen JT, Hunder GG, Goronzy JJ, Warrington KJ, et al. Clinical and pathological evolution of giant cell arteritis: a prospective study of follow-up temporal artery biopsies in 40 treated patients. Mod Pathol. (2017) 30:788-96. doi: 10.1038/modpathol. 2017.10

8. Kermani TA, Warrington KJ. Prognosis and monitoring of giant cell arteritis and associated complications. Expert Rev Clin Immunol. (2018) 14:37988. doi: 10.1080/1744666X.2018.1467758

9. Pfeil A, Oelzner P, Hellmann P. The treatment of giant cell arteritis in different clinical settings. Front Immunol. (2018) 9:3129. doi: 10.3389/fimmu.2018.03129

10. Villiger PM, Adler S, Kuchen S, Wermelinger F, Dan D, Fiege V, et al. Tocilizumab for induction and maintenance of remission in giant cell arteritis: a phase 2, randomised, double-blind, placebocontrolled trial. Lancet. (2016) 387:1921-7. doi: 10.1016/S0140-6736(16) 00560-2

11. Stone JH, Tuckwell K, Dimonaco S, Klearman M, Aringer M, Blockmans D, et al. Trial of tocilizumab in giant-cell arteritis. N Engl J Med. (2017) 377:317-28. doi: 10.1056/NEJMoa1613849

12. Reichenbach S, Adler S, Bonel H, Cullmann JL, Kuchen S, Butikofer L, et al. Magnetic resonance angiography in giant cell arteritis: results of a randomized controlled trial of tocilizumab in giant cell arteritis. Rheumatology. (2018) 57:982-6. doi: 10.1093/rheumatology/key015

13. Spiera RF, Mitnick HJ, Kupersmith M, Richmond M, Spiera H, Peterson MG, et al. A prospective, double-blind, randomized, placebo controlled trial of methotrexate in the treatment of giant cell arteritis (GCA). Clin Exp Rheumatol. (2001) 19:495-501.

14. Hocevar A, Jese R, Rotar Z, Tomsic M. Does leflunomide have a role in giant cell arteritis? An open-label study. Clin Rheumatol. (2019) 38:2916. doi: 10.1007/s10067-018-4232-x

15. Restuccia G, Boiardi L, Cavazza A, Catanoso M, Macchioni P, Muratore F, et al. Flares in biopsy-proven giant cell arteritis in northern italy: characteristics and predictors in a long-term followup study. Medicine. (2016) 95:e3524. doi: 10.1097/MD.00000000000 03524

16. Alba MA, Garcia-Martinez A, Prieto-Gonzalez S, Tavera-Bahillo I, CorberaBellalta M, Planas-Rigol E, et al. Relapses in patients with giant cell arteritis: prevalence, characteristics, and associated clinical findings in a longitudinally followed cohort of 106 patients. Medicine. (2014) 93:194201. doi: 10.1097/MD.0000000000000033

17. Stone JH, Tuckwell K, Dimonaco S, Klearman M, Aringer M, Blockmans $\mathrm{D}$, et al. Glucocorticoid dosages and acute-phase reactant levels at giant cell arteritis flare in a randomized trial of tocilizumab. Arthritis Rheumatol. (2019) 71:1329-38. doi: 10.1002/art.40876

18. Gloor AD, Yerly D, Adler S, Reichenbach S, Kuchen S, Seitz M, et al. Immuno-monitoring reveals an extended subclinical disease activity in tocilizumab-treated giant cell arteritis. Rheumatology. (2018) 57:1795801. doi: 10.1093/rheumatology/key158

19. van Sleen Y, Graver JC, Abdulahad WH, van der Geest KSM, Boots AMH, Sandovici M, et al. Leukocyte dynamics reveal a persistent myeloid dominance in giant cell arteritis and polymyalgia rheumatica. Front Immunol. (2019) 10:1981. doi: 10.3389/fimmu.2019.01981

20. Nadkarni S, Dalli J, Hollywood J, Mason JC, Dasgupta B, Perretti M. Investigational analysis reveals a potential role for neutrophils in giant-cell arteritis disease progression. Circ Res. (2014) 114:242-8. doi: 10.1161/CIRCRESAHA.114. 301374

21. Ivetic A. A head-to-tail view of L-selectin and its impact on neutrophil behaviour. Cell Tissue Res. (2018) 371:43753. doi: 10.1007/s00441-017-2774-x

22. Burja B, Feichtinger J, Lakota K, Thallinger GG, Sodin-Semrl S, Kuret $\mathrm{T}$, et al. Utility of serological biomarkers for giant cell arteritis in a large cohort of treatment-naive patients. Clin Rheumatol. (2019) 38:31729. doi: 10.1007/s10067-018-4240-x

23. Serling-Boyd N, Stone JH. Recent advances in the diagnosis and management of giant cell arteritis. Curr Opin Rheumatol. (2020) 32:201-7. doi: 10.1097/BOR.0000000000000700
24. Dartevel A, Toussaint B, Trocme C, Arnaud M, Simon N, Faure P, et al. Serum amyloid A as a marker of disease activity in giant cell arteritis. Autoimmun Rev. (2020) 19:102428. doi: 10.1016/j.autrev.2019.102428

25. Hunder GG, Bloch DA, Michel BA, Stevens MB, Arend WP, Calabrese LH, et al. The American college of rheumatology 1990 criteria for the classification of giant cell arteritis. Arthritis Rheum. (1990) 33:11228. doi: 10.1002/art.1780330810

26. Mukhtyar C, Guillevin L, Cid MC, Dasgupta B, de Groot K, Gross $\mathrm{W}$, et al. EULAR recommendations for the management of large vessel vasculitis. Ann Rheum Dis. (2009) 68:318-23. doi: 10.1136/ard.2008. 088351

27. van der Geest KS, Abdulahad WH, Chalan P, Rutgers A, Horst G, Huitema MG, et al. Disturbed B cell homeostasis in newly diagnosed giant cell arteritis and polymyalgia rheumatica. Arthritis Rheumatol. (2014) 66:192738. doi: 10.1002/art.38625

28. Ronchetti S, Ricci E, Migliorati G, Gentili M, Riccardi C. How glucocorticoids affect the neutrophil life. Int J Mol Sci. (2018) 19:4090. doi: 10.3390/ijms 19124090

29. Breedveld FC, Dayer JM. Leflunomide: mode of action in the treatment of rheumatoid arthritis. Ann Rheum Dis. (2000) 59:841-9. doi: 10.1136/ard.59.11.841

30. van Sleen Y, Sandovici M, Abdulahad WH, Bijzet J, van der Geest KSM, Boots AMH, et al. Markers of angiogenesis and macrophage products for predicting disease course and monitoring vascular inflammation in giant cell arteritis. Rheumatology. (2019) 58:1383-92. doi: 10.1093/rheumatology/ kez034

31. van Sleen Y, Jiemy WF, Pringle S, van der Geest KSM, Abdulahad WH, Sandovici M, et al. A distinct macrophage subset mediating tissue destruction and neovascularization in giant cell arteritis: implication of the YKL40/Interleukin-13 receptor alpha2 axis. Arthritis Rheumatol. (2021) 73:232737. doi: 10.1002/art.41887

32. Libreros S, Iragavarapu-Charyulu V. YKL-40/CHI3L1 drives inflammation on the road of tumor progression. J Leukoc Biol. (2015) 98:9316. doi: 10.1189/jlb.3VMR0415-142R

33. Kunz LI, van't Wout EF, van Schadewijk A, Postma DS, Kerstjens HA, Sterk PJ, et al. Regulation of YKL-40 expression by corticosteroids: effect on proinflammatory macrophages in vitro and its modulation in COPD in vivo. Respir Res. (2015) 16:154. doi: 10.1186/s12931-015-0314-3

34. Esen I, Jiemy WF, van Sleen Y, van der Geest KSM, Sandovici M, Heeringa $\mathrm{P}$, et al. Functionally heterogenous macrophage subsets in the pathogenesis of giant cell arteritis: novel targets for disease monitoring and treatment. J Clin Med. (2021) 10:4958. doi: 10.3390/jcm10214958

35. Samson M, Bonnotte B. Analysis of IL-6 measurement in patients with GCA treated with tocilizumab should consider concomitant treatment with prednisone. Ann Rheum Dis. (2020) 79:e102. doi: 10.1136/annrheumdis-2019-215697

36. Conway R, O'Neill L, McCarthy GM, Murphy CC, Fabre A, Kennedy S, et al. Interleukin 12 and interleukin 23 play key pathogenic roles in inflammatory and proliferative pathways in giant cell arteritis. Ann Rheum Dis. (2018) 77:1815-24. doi: 10.1136/annrheumdis-2018-213488

37. Miossec P, Kolls JK. Targeting IL-17 and TH17 cells in chronic inflammation. Nat Rev Drug Discov. (2012) 11:763-76. doi: 10.1038/nrd3794

38. Chong AS, Rezai K, Gebel HM, Finnegan A, Foster P, Xu $\mathrm{X}$, et al. Effects of leflunomide and other immunosuppressive agents on $\mathrm{T}$ cell proliferation in vitro. Transplantation. (1996) 61:140-5. doi: 10.1097/00007890-199601150-00026

39. Mus AM, Cornelissen F, Asmawidjaja PS, van Hamburg JP, Boon L, Hendriks RW, et al. Interleukin- 23 promotes Th17 differentiation by inhibiting T-bet and FoxP3 and is required for elevation of interleukin-22, but not interleukin21, in autoimmune experimental arthritis. Arthritis Rheum. (2010) 62:104350. doi: $10.1002 /$ art. 27336

40. Umezawa N, Kawahata K, Mizoguchi F, Kimura N, Yoshihashi-Nakazato Y, Miyasaka N, et al. Interleukin-23 as a therapeutic target for inflammatory myopathy. Sci Rep. (2018) 8:5498. doi: 10.1038/s41598-018-23539-4

41. Espigol-Frigole G, Planas-Rigol E, Lozano E, Corbera-Bellalta M, TerradesGarcia N, Prieto-Gonzalez S, et al. Expression and function of IL12/23 related cytokine subunits (p35, p40, and p19) in giant-cell arteritis lesions: 
contribution of $\mathrm{p} 40$ to Th1- and Th17-mediated inflammatory pathways. Front Immunol. (2018) 9:809. doi: 10.3389/fimmu.2018.00809

Conflict of Interest: MF-B is employed by the company BioMed X Institute, Heidelberg, Germany.

The remaining authors declare that the research was conducted in the absence of any commercial or financial relationships that could be construed as a potential conflict of interest.

Publisher's Note: All claims expressed in this article are solely those of the authors and do not necessarily represent those of their affiliated organizations, or those of the publisher, the editors and the reviewers. Any product that may be evaluated in this article, or claim that may be made by its manufacturer, is not guaranteed or endorsed by the publisher.

Copyright (๑) 2022 Kuret, Frank-Bertoncelj, Lakota, Žigon, Thallinger, Kopitar, Čučnik, Tomšič, Hočevar and Sodin-Šemrl. This is an open-access article distributed under the terms of the Creative Commons Attribution License (CC BY). The use, distribution or reproduction in other forums is permitted, provided the original author(s) and the copyright owner(s) are credited and that the original publication in this journal is cited, in accordance with accepted academic practice. No use, distribution or reproduction is permitted which does not comply with these terms. 